\title{
A Nursing Education Research Framework for Transformative Learning and \\ Interdependence of Academia and Practice
}

Jacinthe Pepin, RN, Ph.D., Professor and Director of the Center for Innovation in Nursing Education (CIFI), Faculty of Nursing, Université de Montréal, Montréal, Canada.

Johanne Goudreau, RN, Ph.D., Professor and Vice-dean, undergraduate and continuing education programs, Faculty of Nursing, Université de Montréal, Montréal, Canada.

Patrick Lavoie, RN, Ph.D., Postdoctoral Fellow, William F. Connell School of Nursing, Boston College, United States.

Marilou Bélisle, Ph.D., Assistant Professor, Faculty of Education, Université de Sherbrooke, Sherbrooke, Canada.

Amélie Blanchet Garneau, RN, Ph.D., Assistant Professor, Faculty of Nursing, Université de Montréal, Montréal, Canada.

Louise Boyer, RN, Ph.D., Assistant Professor, Faculty of Nursing, Université de Montréal, Montréal, Canada.

Caroline Larue, RN, Ph.D., Professor and Vice-dean, graduate programs, Faculty of Nursing, Université de Montréal, Montréal, Canada.

Kathleen Lechasseur, RN, Ph.D., Associate Professor and Vice-dean, undergraduate programs, Faculty of Nursing, Université Laval, Québec, Canada.

Corresponding author: Jacinthe Pepin, jacinthe.pepin@umontreal.ca, C.P. 6128 succ. CentreVille, Montreal, QC, Canada, H3C 3J7

Coauthors: johanne.goudreau@umontreal.ca, patrick.lavoie.1@umontreal.ca, marilou.e.beliste@usherbrooke.ca, amelie.blanchet.garneau @umontreal.ca, louise.boyer@umontreal.ca, caroline.larue@umontreal.ca, kathleen.lechasseur@fsi.ulaval.ca

This paper was written by the Équipe FUTUR, a research team funded with an infrastructure grant from the Fonds de recherché du Québec - Société et culture (FRQSC). 


\section{A nursing education research framework for transformative learning and interdependence of academia and practice}

International experts have called for a transformation of nurses and other health professionals' education to prepare new graduates for evolving and complex healthcare environments (Benner, Sutphen, Leonard, \& Day, 2010; Frenk \& al., 2010). Previous educational reforms focused on the integration of scientific knowledge into university curricula and on pedagogical strategies, such as problem-based learning. Today, what is proposed is a reform to consider the connections between education and healthcare systems to enhance their responsiveness to students' and populations' needs and to improve new graduates' performance. The intent is to prepare future health professionals to provide high quality care in challenging environments so they become active change agents in healthcare systems. This entails that students and graduates become competent to mobilize knowledge in specific contexts, taking into account the peculiarities of their own practice environment, while remaining connected to global systems of healthcare.

Transformative learning and interdependence between academia and practice are strategies recommended by a Lancet commission on education of health professionals to promote quality care and health systems (Frenk \& al., 2010). Transformative learning, defined as a process of transforming one's frames of reference to make them more inclusive, capable of change, and reflective in order to guide action (Mezirow, 2000), goes beyond informative learning, i.e., learning of knowledge and skills, and formative learning, i.e., learning of professional values. Transformative learning is about the development of leadership attributes in future professionals who are to act as change agents. Desirable outcomes include critical thinking, effective teamwork, and adaptation of global resources to local priorities. Interdependence is about how different components of a system interact. For health professionals' education, interdependence implies that academic and practice milieus have to share their practices, resources, and innovations and partner to harmonize professionals' learning trajectory. These recommendations are directed towards a patient-centered perspective with a vision of patient safety and high-quality care.

Questions arise about how nursing education research can contribute to this reform. Nursing education research has had and continues contributing knowledge on educational innovations to ensure better preparation of new graduates. However, a concerted effort is needed to share results and mobilize knowledge in a constantly changing world. This paper presents a contemporary trifold 
nursing education research framework developed for such purpose. This research framework is presented as a contribution to address the pressing issue of "preparing health professionals for a new century by transforming education to strengthen health systems in an interdependent world" (Frenk et al., 2010, p.1923).

This nursing education research framework is grounded in a constructivist competency-based approach (CCBA) to education as a conceptual perspective for students' transformative learning. After describing the CCBA, we will delineate a previous research program, which served to define the three pillars of this overarching research framework: 1) collaborative delineation of nursing education outcomes, 2) clarification of how and why educational interventions contributes to the development of competencies and changes practices, and 3) identification of nursing education sensitive patient and system outcomes. These pillars are aligned with the imperative of interdependence between academic and practice milieus.

\section{Conceptual perspective: A constructivist competency-based approach to education}

The conceptual perspective of this research framework is based on a CCBA to nursing education (Goudreau et al., 2009). CCBA defines the outcomes of nursing education as competencies, which are viewed as complex knowledge of how to act in a variety of situations. Competencies are the manifestation of the mobilization and combination of a diversity of resources, e.g., knowledge, skills, or values necessary for competent nursing practice (Tardif, 2006), acquired through various educational situations and social interactions with patients, peers, and educators. Competencies are developmental and infinite; they continue to build up and mature throughout a learner's lifetime.

While it is concerned with the acquisition of resources and the development of competencies, CCBA also deals with how competencies are to be used in specific professional situations that are to be encountered in various healthcare milieus. Therefore, CCBA aims at transformative learning by emphasising the contextualization of learning. To do so, education builds on situation-based group learning strategies that are designed to give students insights into their future professional practice. Because students work in small groups and are immersed in lifelike professional situations throughout their curriculum, it is expected that they will build critical thinking and collaborative working assets that will be reinvested once they graduate. By doing so, students 
become sensitive to the nature and practice of clinical leadership and learn to be leaders in their own professional practice.

\section{A first research program on CCBA to nursing education}

With a previous grant from the Canadian Foundation for Healthcare Improvement (CFHI, 200812), we conducted a research program grounded in the CCBA. At that time, evidence was cumulating and clinicians had observed that newly graduated nurses experienced difficulties in clinical reasoning and clinical leadership when they entered the workplace. In order to better support the development of those competencies for newly graduated nurses, our faculty and its affiliated teaching hospitals partnered to adapt both parties' educational practices in light of a CCBA. This led to four research studies.

The first two studies were meant for both parties to gain mutual understanding of how nurses' competencies developed in initial training, but also when they entered the workplace. These qualitative studies yielded cognitive learning models of the clinical reasoning and clinical leadership competencies. The clinical reasoning cognitive learning model (Goudreau et al., 2014) described how nurses learned and gained expertise in collecting, analysing, and interpreting assessment data in order to comprehend patients' health experiences. The clinical leadership cognitive learning model (Pepin et al., 2011) depicted nurses' growing sphere of influence, from the integration of leadership actions in their everyday practice to influence on the organizational level and beyond.

Complementary to those studies and coherently with the CCBA, the third study addressed personal and organizational factors that promoted the development of clinical reasoning and clinical leadership when newly graduated nurses entered the workplace (Larue et al., 2013). The results indicated that newly graduated nurses preferred to learn by reflecting with colleagues on clinical situations encountered in clinical practice. However, the lack of space and time for reflection in the workplace was considered a major issue that could impede nurses' competencies development.

With this knowledge in hand, clinical and academic partners worked together to develop and implement an educational intervention to support the development of newly graduated nurses' 
competencies, in the workplace. The three previous studies of the research program culminated in the creation of a series of 30-minute group reflections on clinical situations that newly graduated nurses encountered in their clinical practice. The group reflections were implemented in the research program's fourth study (Goudreau et al., 2015). Its evaluation showed that a continuing education intervention aligned with the CCBA to nursing education had the potential to contribute to the development of nurses' clinical reasoning and clinical leadership.

Overall, these studies embodied a CCBA to promote transformative learning of newly graduated nurses. Furthermore, they fitted in a vision of interdependence between academic and practice milieus. Partnership between academic and practice milieus resulted in a shared understanding of the development of newly graduated nurses' competencies. This allowed for a joint effort towards the development and evaluation of an educational intervention tailored for newly graduated nurses entering the workplace. As it was aligned with their level of development and learning needs, the fourth study revealed that newly graduated nurses, more experienced clinicians, and managers perceived the benefits of the educational intervention. Nevertheless, further work and reflection were needed to contribute evidence that this and other educational interventions could really have an impact on nursing practice and healthcare systems. This is how we came to define three pillars of a contemporary nursing education research framework.

\section{Three pillars of a contemporary nursing education research framework}

To pursue the advancement of nursing education research for interdependence and transformative learning, we proposed a trifold research framework. As suggested by Cook et al. (2008), this framework posits that research on educational innovations should elucidate how and why they contribute to students' learning and other outcomes.

The first pillar is to foster collaboration among nursing leaders from educational and clinical settings to define the outcomes of nursing education. As exemplified in the four studies, nursing educators, researchers, and clinicians share a common interest in the best ways to access and deliver care. Nurses and managers from clinical settings are direct witnesses of the evolution of populations' needs. Meanwhile, nursing educators and researchers are concerned with the processes of teaching and learning to be a nurse. Collaboration of educators, clinicians, and researchers, has the potential to transform the conception of what is needed for competent nursing 
in the modern healthcare systems. This entails new conceptions of the desirable outcomes of nursing education in terms of global competencies for clinical practice. Those conceptions need to rely on empirical studies of how competencies develop in nurses and what are their critical learning milestones. In that way, clinical nursing can remain responsive to the evolution of patients' needs and participate in transforming health systems. Nursing education research can produce learning models of how one becomes a competent nurse, as show in the two first studies.

When competencies in nursing practice are defined, their development shall be positioned on a continuum that begins in the academic setting and continues in practice milieus. After undergraduate education, professional expertise continues to develop within clinical practice. Therefore, academic and practice milieus become two interrelated settings for nurses' ongoing learning throughout their professional life. Hence, both milieus can collaborate to plan educational efforts that are coherent with the learning models that have been developed. The second pillar focuses on the clarification of how and why educational interventions contributes to the development of competencies and therefore to the preparation of competent nurses. CCBA educational interventions engage nurses in the process of continuous learning and application in practice. This pillar aims at capturing through research the learning processes and the change that competencies development produces in practice, in various contexts. Knowledge development on innovative educational strategies that foster transformative learning in both academic and clinical settings is an important contribution of nursing education research.

The third pillar consists of identifying nursing education sensitive patient and system outcomes. An interdependent approach to nursing education implies that evaluation of the impact of cuttingedge educational practices goes beyond nursing competencies and reaches towards indicators of patient safety, quality of care, and care environments. Much work on this matter is needed given the complexity of healthcare systems and how they produce certain patient outcomes. However, collaboration between education, clinical, and health services researchers provides a greater variety of research tools and indicators to evaluate the impact of educational efforts. This work is necessary in order to understand how equipping nurses with the "right" competencies contribute to safety and quality care and strengthen health systems.

\section{Further development}


The three research pillars, i.e., 1) collaborative delineation of nursing education outcomes, 2) clarification of how and why educational interventions contributes to the development of competencies and changes practices, and 3) identification of nursing education sensitive patient and system outcomes, set the course to contribute to the science of nursing education. Each pillar requires intra- and inter-disciplinary collaborations if implementing the research framework is to produce knowledge useful to both education and clinical milieus. The beginning of our research program exemplified interdependence between academic and practice milieus towards transformative learning. Our ongoing research program, conducted with an interdisciplinary team (nursing and education sciences), now focusses on elucidating how and why CCBA educational interventions contribute to nursing students' learning. The nursing education research framework serves as the theoretical and methodological orientations of this program, which is supported by a research team infrastructure grant from the Fonds de recherche du Québec - Société et Culture (FRQSC, 2013-2017).

Further work is needed to understand the impact of educational interventions on patient and systems outcomes. To do so, researchers from the clinical and educational domains have partnered within the Quebec Nursing Intervention Research Network (Fonds de recherche du Québec - Santé, 2012-2019). This network focuses on the development, implementation, and evaluation of nursing interventions in the clinical, educational, and organizational domains. With the joint expertise of seasoned researchers from different background, we hope to identify outcomes of educational innovations that are aligned with the proposed reform for nurses and other health professionals' education. The ongoing research programs bring us closer to transformative learning and to interdependence between academic and practice milieus in nursing education. Reinforcing collaborative engagement between academic and practice milieus is a necessity as both contexts are evolving and increasingly challenging. Nursing education research could strengthen its knowledge contribution and the difference it makes in clinical practice by seeking both educational and clinical outcomes.

\section{References}

Benner, P., Sutphen, M., Leonard, V., \& Day, L. (2010). Educating nurses: A call for radical transformation. San Francisco, CA: Jossey-Bass. 
Cook, D. A., Bordage, G., \& Schmidt, H. G. (2008). Description, justification and clarification: a framework for classifying the purposes of research in medical education. Medical Education, 42(2), 128-133. https://doi.org/10.1111/j.1365-2923.2007.02974.x

Frenk, J., Chen, L., Bhutta, Z. A., Cohen, J., Crisp, N., Evans, T., ... Zurayk, H. (2010). Health professionals for a new century: Transforming education to strengthen health systems in an interdependent world. The Lancet, 376(9756), 1923-1958. https://doi.org/10.1016/S0140$\underline{6736(10) 61854-5}$

Goudreau, J., Pepin, J., Dubois, S., Boyer, L., Larue, C., \& Legault, A. (2009). A second generation of the competency-based approach to nursing education. International Journal of Nursing Education Scholarship, 6(1). https://doi.org/10.2202/1548-923X.1685

Goudreau, J., Boyer, L., \& Létourneau, D. (2014). Clinical nursing reasoning in nursing practice: A cognitive learning model based on a think aloud methodology. Quality Advancement in Nursing Education-Avancées en formation infirmière, 1(1), 4. https://doi.org/10.17483/2368-6669.1009

Goudreau, J., Pepin, J., Larue, C., Dubois, S., Descôteaux, R., Lavoie, P. et Dumont, K. (2015). A competency-based approach to continuing education: Implementing a series of 30minute reflective practice sessions on eight units of care. Nurse Education in Practice, 15, 572-578. https://doi.org/10.1016/j.nepr.2015.10.013

Larue, C., Dubois, S., Girard, F., Goudreau, J., \& Dumont, K. (2013). Le développement continu des compétences de raisonnement clinique et de leadership: Facteurs personnels et facteurs organisationnels. Recherche en soins infirmiers, (1), 76-87.

Mezirow J. (2000). Learning as transformation: critical perspectives on a theory in progress. San Francisco, CA: Jossey Bass.

Pepin, J., Dubois, S., Girard, F., Tardif, J., \& Ha, L. (2011). A cognitive learning model of clinical nursing leadership. Nurse Education Today, 31(3), 268-273. https://doi.org/10.1016/j.nedt.2010.11.009

Tardif, J. (2006). L'évaluation des compétences. Documenter le parcours de développement. Montréal : Chenelière Éducation. 